Research Article

\title{
Feature Extraction Method for Weak Faults Based on Time-Delayed Feedback Mixed Potential Stochastic Resonance
}

\author{
Jiachen Tang, ${ }^{1,2}$ Boqiang Shi $\mathbb{D}^{1}{ }^{1}$ and Zhixing $\mathrm{Li}^{1}$ \\ ${ }^{1}$ Department of Mechanical Engineering, University of Science and Technology Beijing, Beijing 100083, China \\ ${ }^{2}$ Department of Mechanical and Industrial Engineering, University of Toronto, Toronto, ON M5S 3G8, Canada \\ Correspondence should be addressed to Boqiang Shi; shiboqiang@ustb.edu.cn
}

Received 7 December 2019; Revised 22 February 2020; Accepted 14 May 2020; Published 28 May 2020

Academic Editor: Franck Poisson

Copyright (c) 2020 Jiachen Tang et al. This is an open access article distributed under the Creative Commons Attribution License, which permits unrestricted use, distribution, and reproduction in any medium, provided the original work is properly cited.

To extract weak faults under strong noise, a method for feature extraction of weak faults with time-delayed feedback mixed potential stochastic resonance (TFMSR) is proposed. This method not only overcomes the saturation characteristics of classical bistable stochastic resonance (CBSR), but also verifies a new potential function model. Based on this model, considering the short memory characteristics of the CBSR method, a method is proposed that can add historical information to the negative feedback process of the stochastic resonance (SR). Through the combination of the above two methods, the weak fault extraction under strong background noise is realized. The article analyzes the effects of the delay term, feedback term, and system parameter on the effect of SR and uses the ant colony algorithm (ACA) to optimize the above parameters. Finally, through simulated and engineering experimental results, it is proved that the proposed method has more advantages than the CBSR method in weak fault feature extraction.

\section{Introduction}

With the development of technology, equipment becomes large scale and intelligent with high speed $[1,2]$. Failure of any component in the system can jeopardize the operational safety of the entire device. Therefore, early prediction of weak faults in mechanical systems is particularly important for the extraction of the weak fault feature. It is an emerging science to study how to extract weak signals from strong noise. It requires multiple disciplines to work together, so it is also an interdisciplinary $[3,4]$.

Benzi et al. [5] first proposed SR when explaining the problem of ancient glaciers. It transfers some parts of the noise energy to the weak signal. While enhancing the faint fault characteristics, some noise becomes weakened, thereby improving the signal-to-noise ratio (SNR). Existing noise reduction methods, such as wavelet analysis [6-8], empirical mode decomposition (EMD) [9], variational mode decomposition $[10]$, and dictionary learning $[11,12]$, can weaken the useful weak signal when filtering the noise. As a signal processing tool, SR has an interesting feature; that is, the noise added into the nonlinear system can excite and amplify the early weak fault features. Utilizing this principle, Yang et al. [13] obtained an amazing result. They recover unknown signals completely submerged in strong noise through a new SR method. Due to this unique characteristic of SR, it has attracted wide attention in the fields of physics [14], medicine [15], and machinery.

Initially, SR theory suffers from the small frequency limitation under adiabatic approximate conditions [16]. Therefore, it cannot be applied in actual engineering. In order to make SR universal $[17,18]$, many researchers have made their own contributions. Leng et al. [19] studied a recalibration of the frequency SR method to achieve a large parameter signal SR. Tan et al. [20] used frequency shift variable scale processing SR to alleviate the contradiction between the number of sample points and sampling frequency. Later, the CBSR model became the most widely used SR model. Li et al. [21] proposed a Morlet wavelet transform CBSR method that can obtain a finer frequency, which is used to extract wind turbine fault characteristics. Lei et al. [22] proposed an adaptive SR method for mechanical fault diagnosis. He et al. [23] proposed a new multiscale noise adjustment method to improve SR performance. Lu et al. [24] proposed a multiscale noise adjustment SR method, which was proved in the 
detection of actual sound signals from train bearings. Shi et al. [25] found that feedback parameters, time-delay parameters, and asymmetric parameters all have an effect on SR, and the time-delay parameters are more sensitive. Li and Shi [26] proposed an SR with a bistable constrained potential and applied to weak fault feature extraction of bearings. Nishiguchi and Fujiwara [27] suggested boosting an effect of CBSR and silhouette noise from noise via a field-effect transistor. The above method mainly uses the CBSR method to lay a foundation for the rapid development of SR. Later, Zhao et al. [28] proved the existence of the output saturation characteristics of the CBSR system. Gosak et al. [29] studied the negative effects of output saturation on the system output in an ultradamped oscillator. Zhang et al. [30] studied SR output saturation based on the additive and multiplicative noises. These studies have recognized the effect of output saturation characteristics on SR, but have not proposed an effective solution. When saturation occurs, the amplitude of the output signal will not increase as the amplitude of the input signal increases. This makes it difficult to extract weak fault characteristics. Therefore, this paper proposes a mixed potential SR model to solve the saturation problem of the CBSR model. Further, the CBSR system is also a short-memory system, without considering the effects of time delay and feedback. This makes that the output of the system depend on the output of the previous one. Lv et al. [31,32] studied the special role of delay in nonlinear systems. Zhuo et al. [33, 34] studied the application of memory and feedback models in fault diagnosis. In summary, adding historical information to the system's feedback process in a certain form will greatly improve the output signal-to-noise ratio (SNR).

This paper proposes a method for extracting weak fault features based on TFMSR. This method not only overcomes the saturation characteristics of the CBSR, but also adds historical information to the system's feedback process in a certain form, effectively solving the two major shortcomings of the CBSR method. Through simulated and experimental results, it proves that the proposed method has more advantageous than the CBSR method in weak fault feature extraction.

\section{Theoretical Foundation}

The CBSR model is expressed as

$$
\frac{\mathrm{d} x}{\mathrm{~d} t}=\frac{-\mathrm{d} U_{1}(x)}{\mathrm{d} x}+s(t)+\xi(t)
$$

where $s(t)=A \cos (\Omega t)$ is a weak periodic signal, $A$ is the amplitude, and $\xi(t)$ is a zero mean Gaussian white noise. The following condition is satisfied:

$$
E[\xi(t) \xi(t+\tau)]=2 D \delta(t-\tau),
$$

where $D$ is the noise intensity. The potential function of the CBSR system is expressed as

$$
U_{1}(x)=-\frac{1}{2} a x^{2}+\frac{1}{4} b x^{4}
$$

where $a$ and $b$ represent the system parameters. In Figure 1, $U_{1}(x)$ is the potential function curve of the CBSR model.

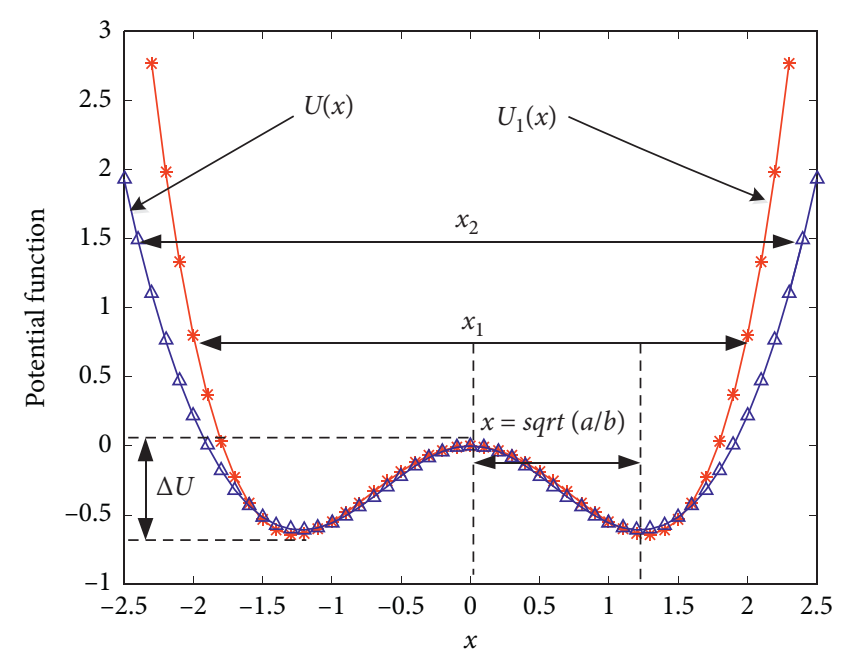

FIgURE 1: The mixed potential function model curve $U(x)$ and the CBSR potential function curve $U_{1}(x)(a=1, b=1)$.

Without loss of generality, we set $a=1$ and $b=1$. Random boost is related to the specific nonlinear form of the potential function. By selecting different nonlinear potential functions and adjusting related parameters, the random boost can be effectively enhanced. Therefore, in order to avoid the output saturation characteristic of the CBSR potential function, we propose a mixed potential stochastic resonance model. It is expressed as

$$
U(x)=\exp \left(-x^{2}\right)-\ln e+\frac{1}{4} m x^{4},
$$

where $m$ is the system parameter. The mixed potential function model curve $U(x)$ and the CBSR potential function curve $U_{1}(x)$ are shown in Figure 1. The potential function of the mixed potential model is wider than that of CBSR, which reflects that the proposed method has more advantages in antisaturation characteristics than CBSR [30].

Considering the effects of the delay term and the feedback term, the Langevin equation is obtained as follows:

$$
\begin{aligned}
& \frac{\mathrm{d} x(t)}{\mathrm{d} t}=-\frac{\mathrm{d} U(x)}{\mathrm{d} x}+K x(t-\sigma)+s(t)+\xi(t) \\
& \frac{\mathrm{d} x(t)}{\mathrm{d} t}=-2 x e^{-x^{2}}-m x^{3}+K x(t-\sigma)+A \cos (\Omega t)+\xi(t) .
\end{aligned}
$$

The probability density of Fokker-Planck with approximate delay feedback can be expressed as [35]

$$
\frac{\partial P(x, t)}{\partial t}=-\frac{\partial\left[h_{n} P(x, t)\right]}{\partial x}+D \frac{\partial^{2} P(x, t)}{\partial x^{2}},
$$

where $h_{n}$ is the conditional average drift. $h_{n}$ can be obtained [36] as

$$
h_{n}=-(1+K \sigma)\left(2 x e^{-x^{2}}+m x^{3}-K x\right)+(1+K \sigma) A \cos (\Omega t) \text {. }
$$

Without considering the effects of periodic signals, equation (7) can be deduced as 


$$
U_{e}(x)=(1+K \sigma)\left(e^{-x^{2}}-\ln e+\frac{m}{4} x^{4}-\frac{K}{2} x^{2}\right) .
$$

Compared with the CBSR, the potential well of TFMSR is affected by the system parameter $m$, the feedback term $K$, and the delay term $\sigma$. As shown in Figure 2, by adjusting the values of $m, K$, and $\sigma$, we can observe that the shape of the potential well is constantly changing.

In Figures 2(a) and 2(b), as the feedback intensity $K$ decreases from 1.1 to 0.7 , the height of the potential barrier remains unchanged, but the bottom of the potential well moves slowly upward. In Figures 2(c) and 2(d), by adjusting the delay term $\sigma$, the depth of the potential well continuously changes. As $\sigma$ increases from 0.4 to 1.2 , the steepness of the potential wall increases. In Figures $2(\mathrm{e})$ and $2(\mathrm{f})$, as $m$ decreases from 1 to 0.6 , the steepness of the potential wall does not change, but the depth of the potential well increases.

According to the theory of the two-state model [37], the probability density of particles moving between two potential wells can be expressed as

$$
R_{ \pm}(t)=\frac{K(1+K \sigma)}{\sqrt{2} \pi} \exp \left(-\frac{K^{2}(1+K \sigma)}{4 D m}\right) \exp \left(\mp \frac{\sqrt{K / m} A(1+K \sigma)}{4 D} \cos \Omega t\right)
$$

Under small delay condition, equation (9) can be deduced as

$$
\lambda=\frac{K(1+K \sigma)}{\sqrt{2} \pi} \exp \left(-\frac{K^{2}(1+K \sigma)}{4 D m}\right) .
$$

Under adiabatic approximation [38], the power spectral density expression is

$$
s(\omega)=s_{1}(\omega)+s_{2}(\omega),
$$

where the output power spectral density of the signal can be expressed as

$$
\begin{aligned}
s_{1}(\omega)= & \frac{K \pi}{2 m}\left(\frac{(1+K \sigma) A \sqrt{K / m}}{D}\right)^{2} \frac{4 \lambda^{2}}{4 \lambda^{2}+\Omega^{2}} \\
& \cdot[\delta(\omega-\Omega)+\delta(\omega+\Omega)] .
\end{aligned}
$$
as

The power spectral density of the noise can be expressed

$$
s_{2}(\omega)=\left[1-\frac{A^{2} K(1+K \sigma)^{2} 4 \lambda^{2}}{2 m D^{2}\left(4 \lambda^{2}+\Omega^{2}\right)}\right] \frac{4 K \lambda}{m\left(4 \lambda^{2}+\omega^{2}\right)} .
$$

The output SNR of the system can be expressed as [39]

$$
\mathrm{SNR}=\frac{\sqrt{2} A^{2} K^{2}}{4 m D^{2}}(1+K \sigma)^{3} \exp \left(-\frac{\left(K^{2}+K^{3} \sigma\right)}{4 m D}\right) .
$$

In Figure 3(a), the system parameter $m$ and delay term $\sigma$ remain unchanged. As the noise intensity $D$ increases, a single peak appears in the output SNR. As analyzed in Section 2, as $K$ decreases, the depth of the potential well is reduced. The energy required for a particle to transition from one potential well to another decreases, the output signal becomes larger, and the effect of SR is obvious. In Figure 3(b), the system parameter $m$ and the feedback intensity $K$ remain unchanged. When $\sigma=0.4$, because $\sigma$ is small, the particles cannot break through the barrier of the potential and realize the transition from one potential well to another. The effect of SR is not obvious. Increasing $\sigma$ to 1.2, the particles have enough energy to cross the potential barrier. At this time, the particles oscillate back and forth between the two potential wells. In Figure 3(b), we can see that the effect of SR is obvious. In Figure 3(c), the feedback intensity $K$ and the delay term $\sigma$ remain unchanged. When the system parameter $m=0.6$, the potential wall is too steep, and the particles cannot obtain sufficient acceleration to break through the barrier constraint. Increasing $m$ to 1 , the potential well depth decreases, and the potential function structure can form the best match with the periodic force, so as to achieve the best SR effect.

\section{Proposed Model}

Through the above analysis, we can conclude that the effect of SR is most obvious when the delay term, feedback intensity, and system parameters are optimally matched. This article uses ACA to match and optimize the above parameters. The detection strategy of the TFMSR method is as follows:

(1) Signal Preprocessing. First, the acquired signal is demodulated by the Hilbert transform, and then the small parameters (with signal amplitude, noise intensity, and signal frequency all being far less than 1) required for SR are obtained by frequency shift variable scale processing [20].

(2) Parameter Initialization and Optimization. First, initialize the parameters and optimize delay term, feedback intensity, and system parameter. The range of parameter optimization is set to $[0,5]$.

(3) Calculating the SNR. The optimal match of parameters are inputted into the SR system. The maximum output SNR of the TFMSR method is calculated by the Runge-Kutta equation [40].

(4) Postprocessing. Through the postprocessing of the signal by the TFMSR system, the faint fault features are extracted. Figure 4 shows the detection strategy of the TFMSR method.

\section{Simulation}

To verify the effort of the TFMSR method, we decided to conduct a simulation experiment. First, a periodic signal with 

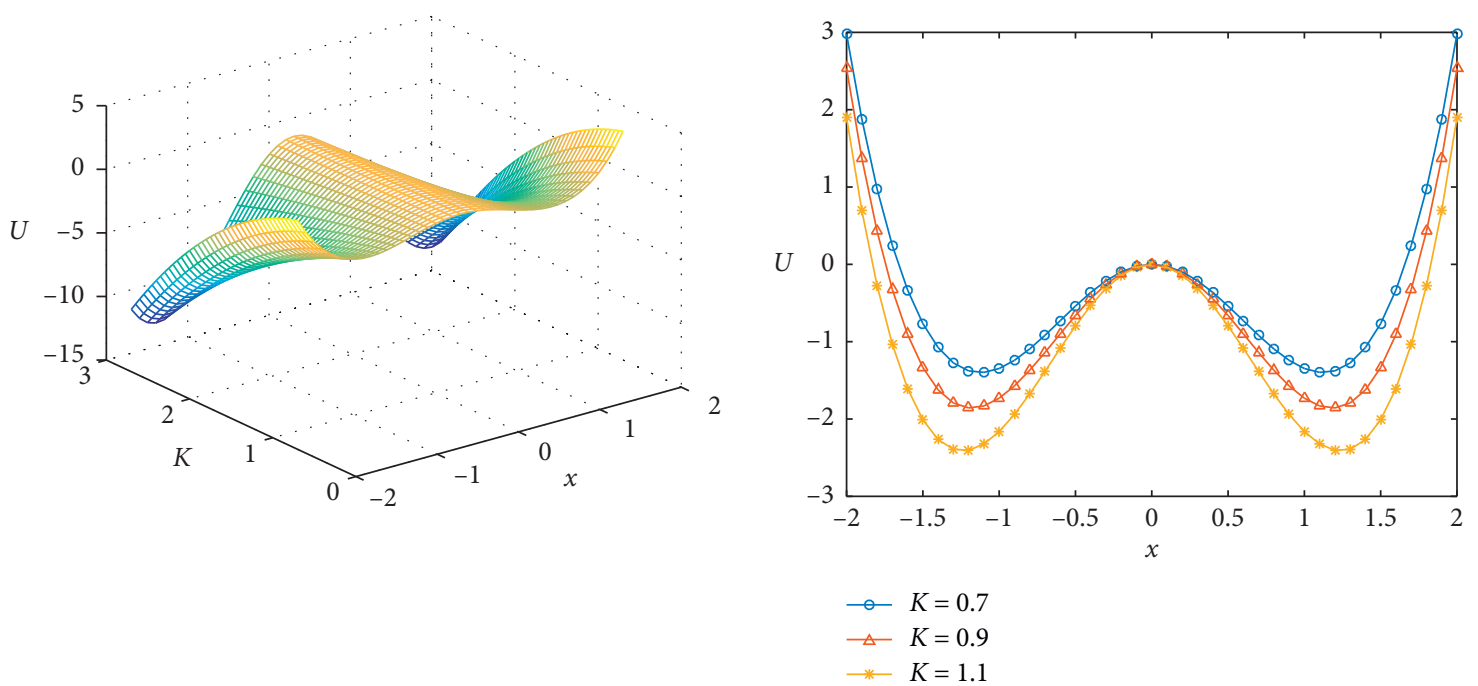

(a)

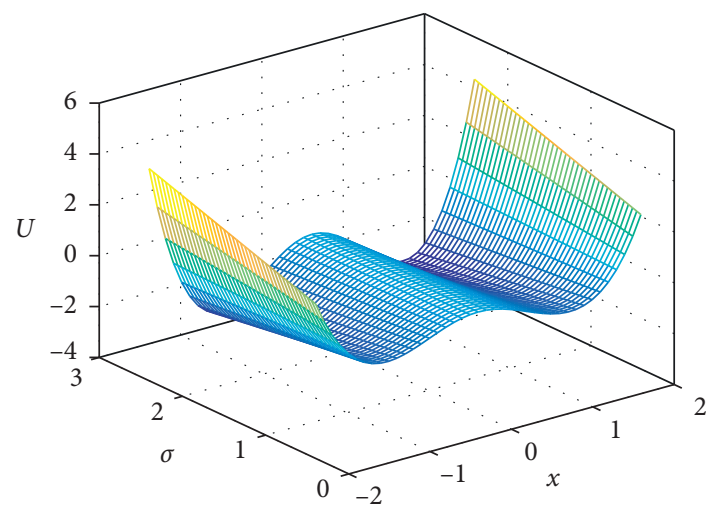

(b)

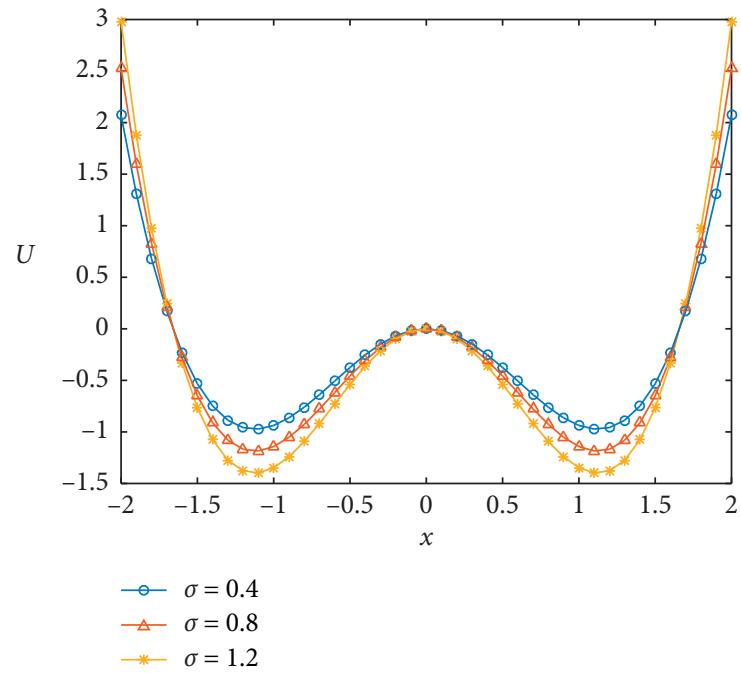

(c)

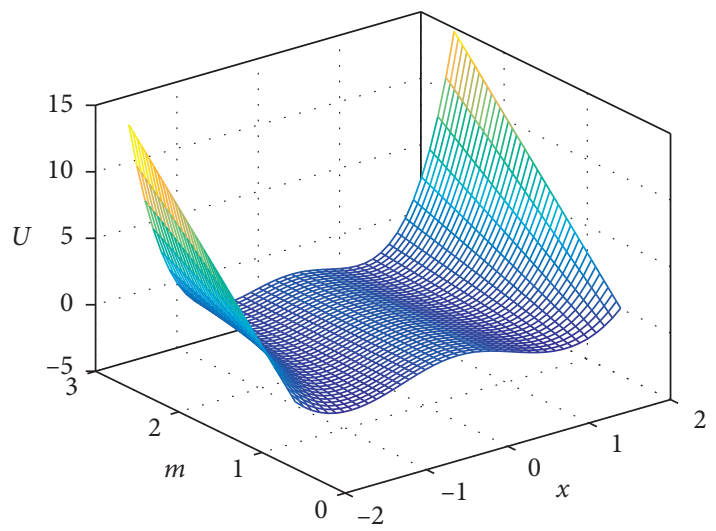

(d)

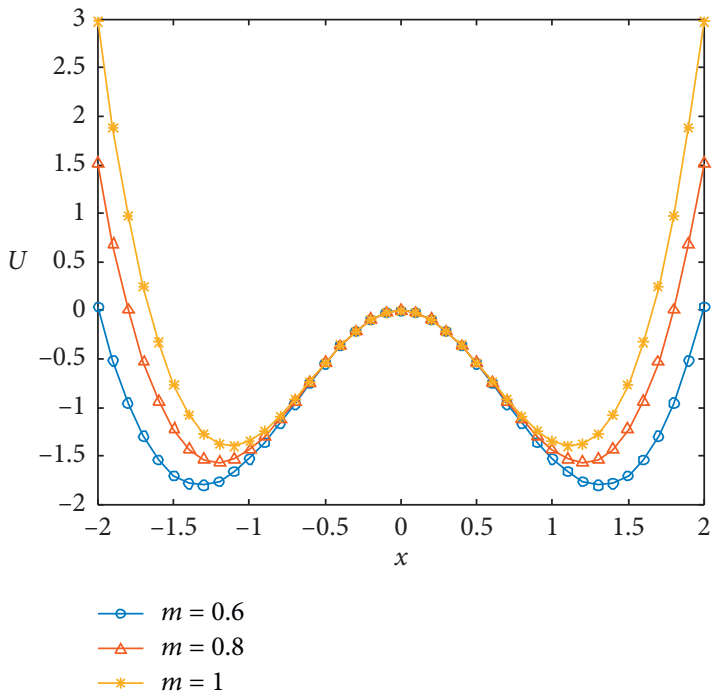

(e)

(f)

Figure 2: Curve of potential function with different parameters: (a) when $m=1$ and $\sigma=1.2$, the relationship between $U$, $x$, and $K$; (b) detailed relationship diagram between $U$ and $K$; (c) when $m=1$ and $K=0.7$, the relationship between $U, x$, and $\sigma$; (d) detailed relationship diagram between $U$ and $\sigma$; (e) when $K=0.7$ and $\sigma=1.2$, the relationship between $U$, $x$, and $m$; (f) detailed relationship diagram between $U$ and $m$. 


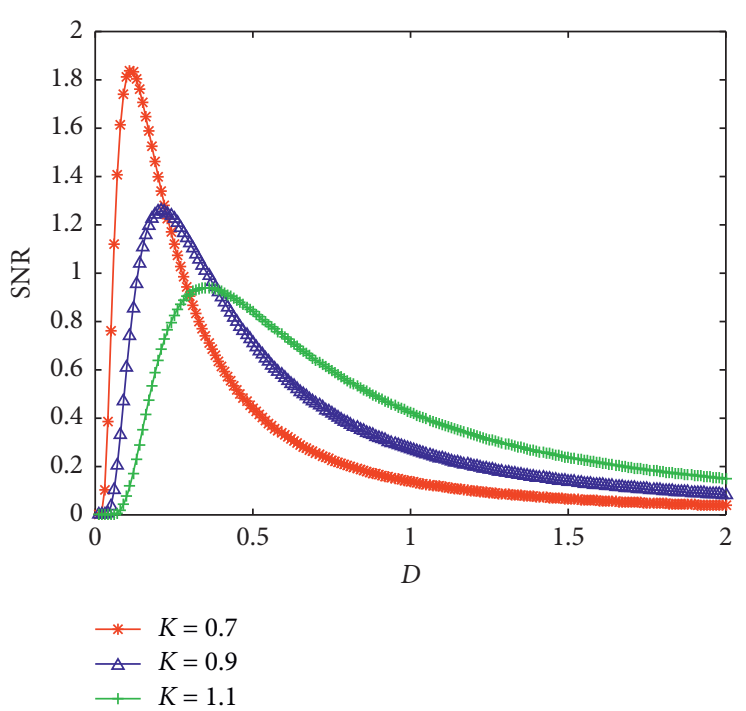

(a)

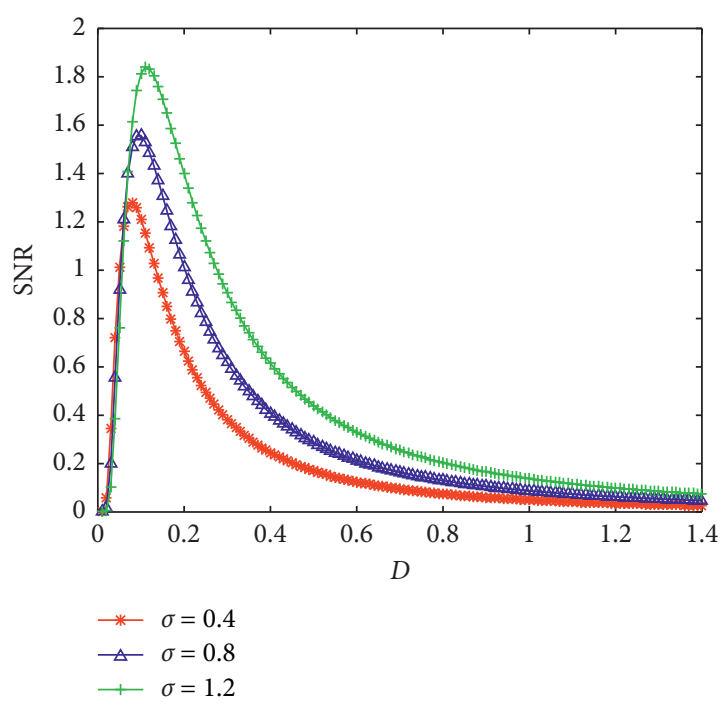

(b)

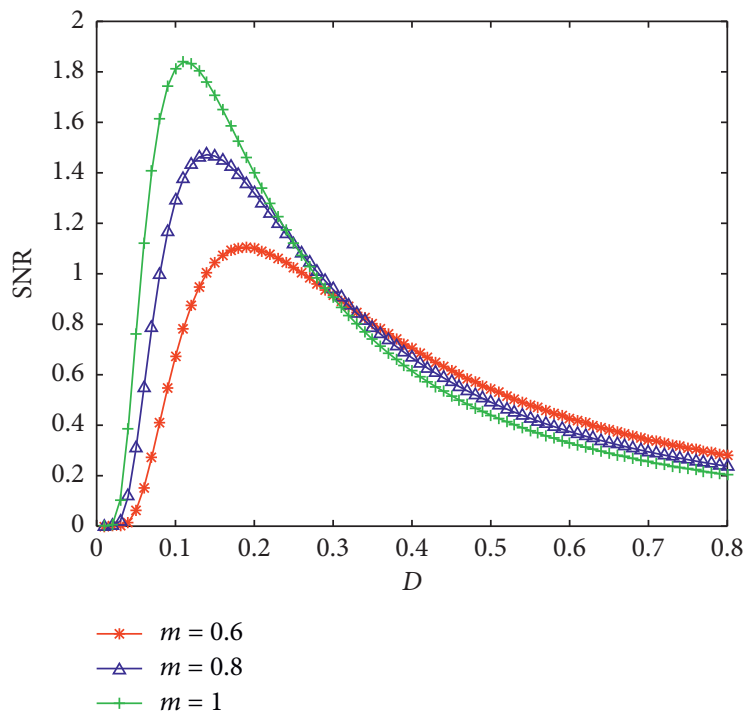

(c)

Figure 3: The change trends of SNR versus D: (a) $m=1$ and $\sigma=1.2$; (b) $m=1$ and $K=0.7$; (c) $K=0.7$ and $\sigma=1.2$.

frequency $f=55 \mathrm{~Hz}$ and amplitude $A=1$ is simulated. The sampling frequency is $10000 \mathrm{~Hz}$. Figures $5(\mathrm{a})-5(\mathrm{c})$ show the time domain, the spectrum, and the envelope spectrum of the simulated signal. Since the background noise is too strong and the characteristic frequency is submerged, we cannot get the fault characteristic frequency. Here, we use the TFMSR method to process the simulated signal. The ACA was used to adaptively optimize the system parameters. The obtained parameters are $\sigma=1.4623, K=0.3321$, and $m=0.9928$. The time domain and spectrum obtained by the simulated signal are shown in Figures 6(a) and 6(b). Figure 6(b) shows that the spectral peak value $A_{\max }$ obtained by using the TFMSR method is 0.07659 . The value of the second highest point is 0.03546 . The difference value $\Delta A$ is 0.04113 . We propose a new evaluation index to evaluate the effectiveness of the two methods. We use $\Delta A / A_{\max }$ to get a dimensionless constant. The value of the dimensionless constant is 0.537 .
For comparison, we will use the CBSR method to process the simulated signal. The initial parameters are settled consistent with the TFMSR method. The obtained parameters are $a=0.0369$ and $b=0.117$. The parameters are substituted into the CBSR system. Figures 6(c) and 6(d) show the time domain and spectrum of the simulated signal. In Figure 6(d), the spectral peak value $A_{1 \max }$ is 0.04329 . The value of the second highest point is 0.03364 . The difference value $\Delta A_{1}$ is 0.00965 . $\Delta A_{1} / A_{1 \max }=0.2229$. In summary, the proposed TFMSR method can enhance weak signals effectively.

\section{Engineering Experiment Verification}

In the field of metallurgy, rolling mills are widely used. Due to the harsh working environment, the fault signals of the components in the equipment are easily overwhelmed by strong noise. Some minor failures, if cannot be found in 


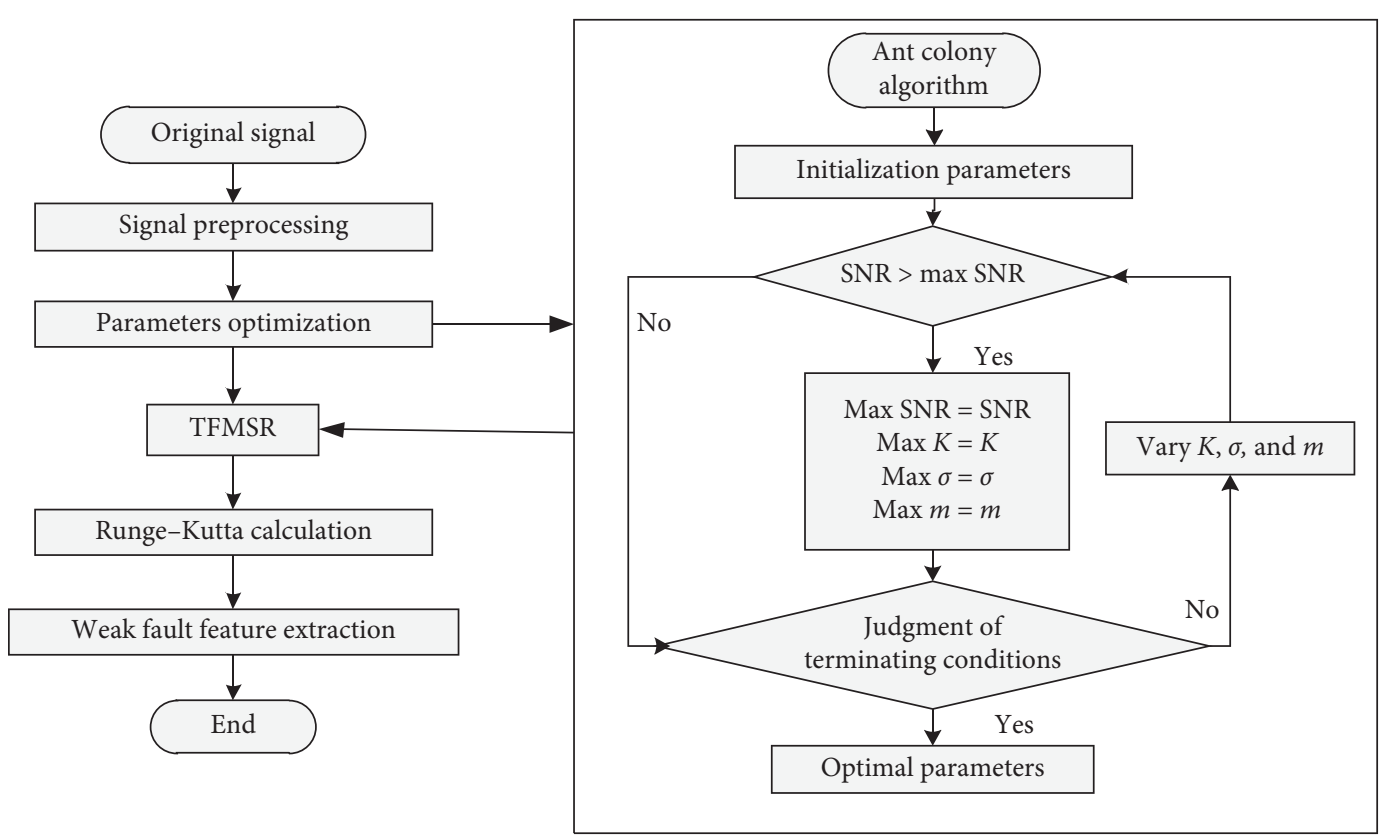

FIGURE 4: TFMSR weak signal detection strategy.

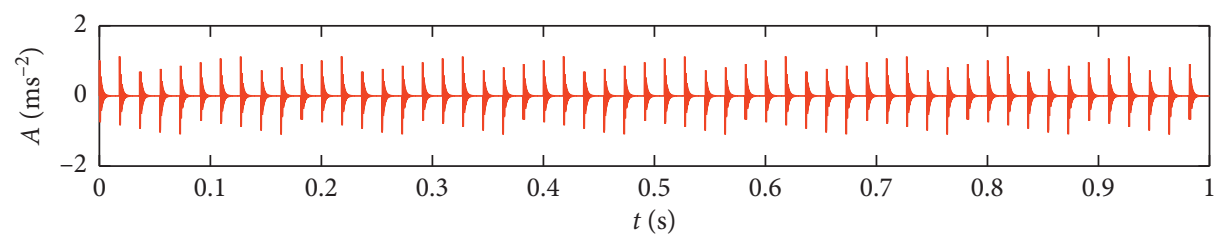

(a)

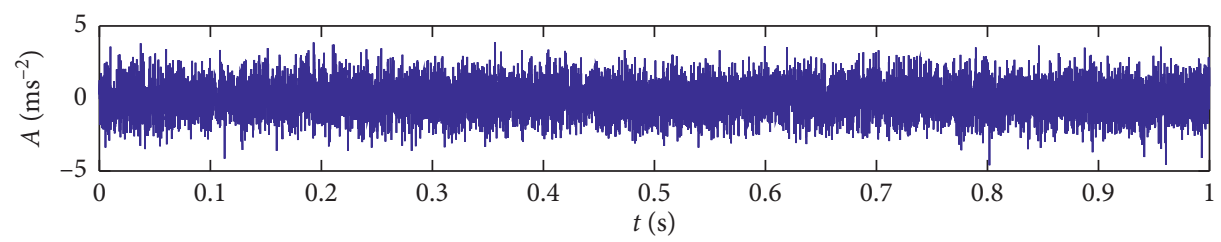

(b)

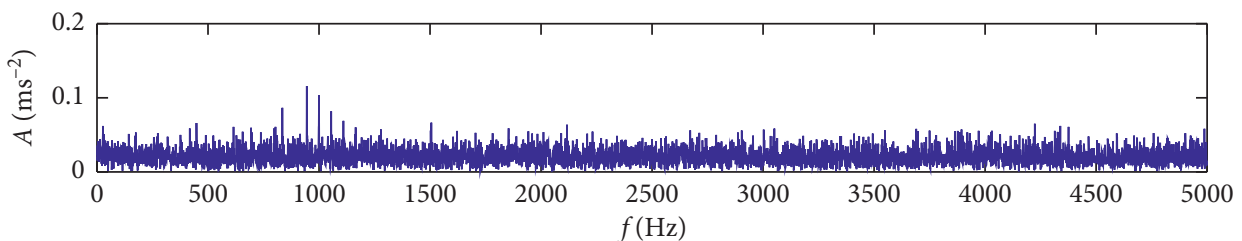

(c)

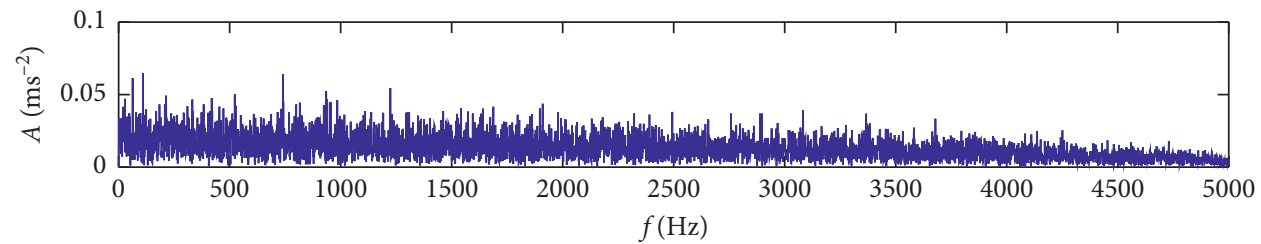

(d)

Figure 5: (a) Simulation signal without noise; (b) simulation signal with noise; (c) spectrum; (d) envelope spectrum. 


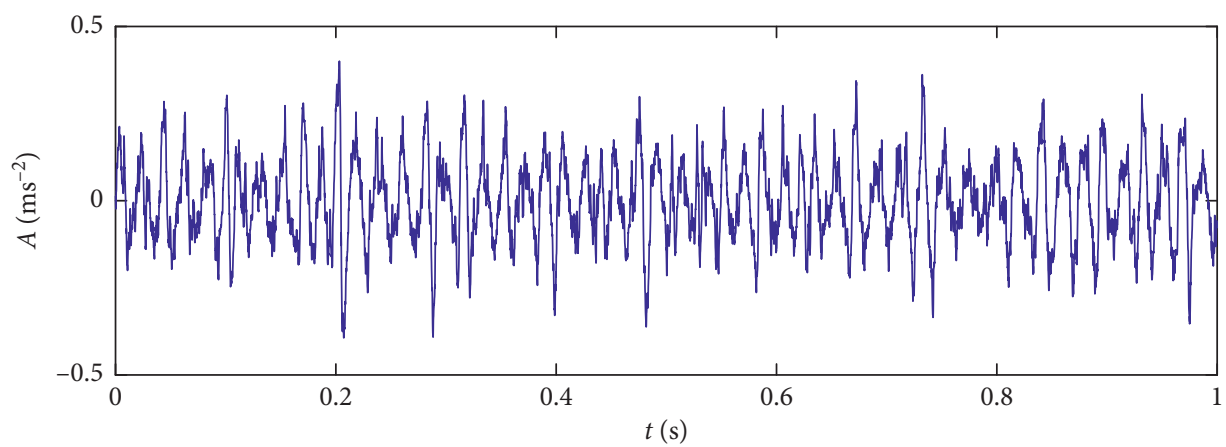

(a)

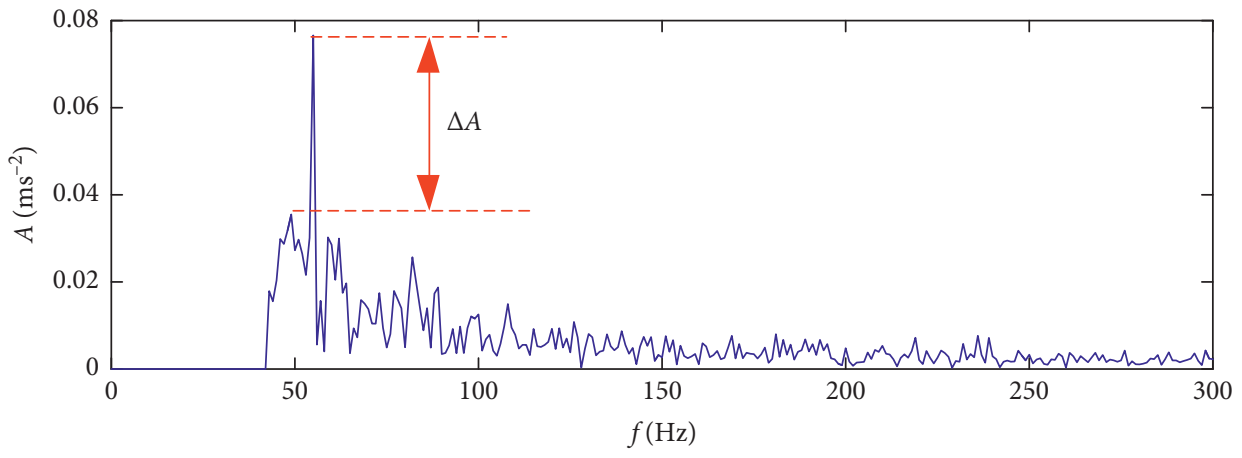

(b)

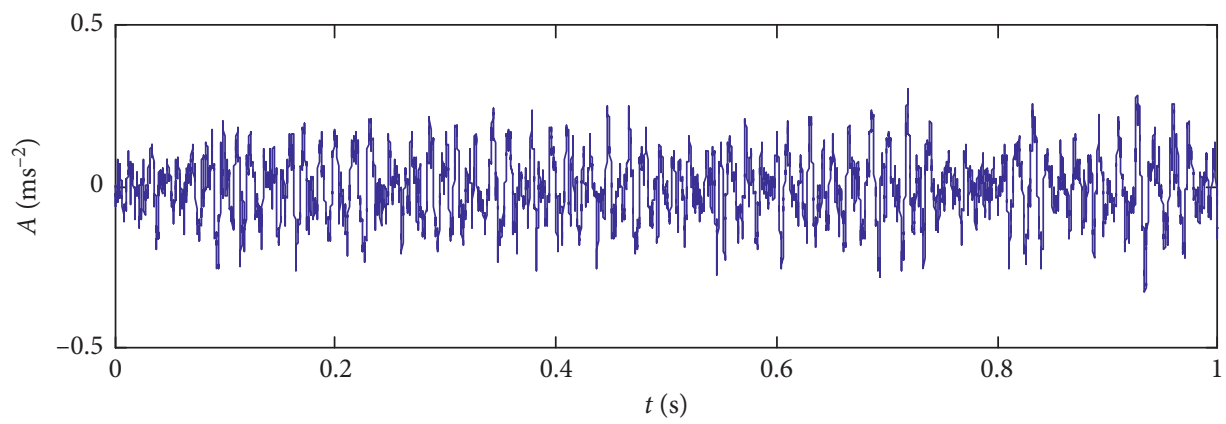

(c)

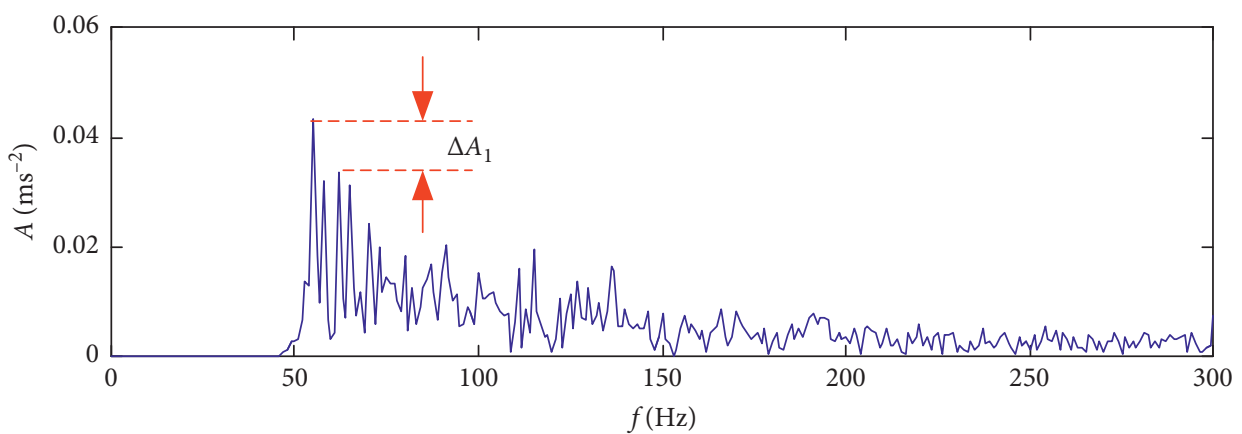

(d)

FIgURE 6: The results of the TFMSR method: (a) time domain waveform of the simulated signal; (b) spectrum. The results of the CBSR method: (c) time domain waveform of the simulated signal; (d) spectrum.

time, may create serious consequences over time. The hot rolling production site of a thin plate factory is shown in Figure 7. The working environment of the equipment is very bad. Through routine inspections, we found that the gearbox of the rolling mill failed, but it was not possible to determine which component was faulty. The on-site signal acquisition device is the ZonicBook/618E, and the sampling position is shown in Figure 8.

Table 1 lists the basic parameters of the gearbox. The acquired signal is processed to obtain the original signal, 


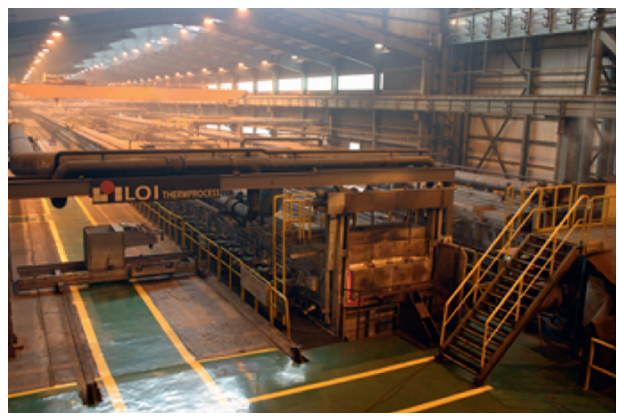

FIgURE 7: Thin-plate mill hot rolling production site.

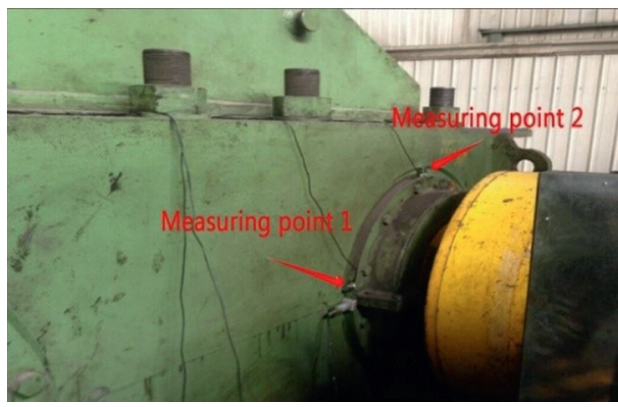

Figure 8: Sensor placement location.

TABle 1: Parameters of the gearbox.

\begin{tabular}{lcccc}
\hline Name & Speed $(\mathrm{r} / \mathrm{min})$ & Parameters & \multicolumn{2}{c}{ Characteristic frequency $(\mathrm{Hz})$} \\
\hline$Z_{1}$ & 63 & 32 & 33.6 \\
$Z_{2}$ & 20.5714 & 98 & 33.6 & 1.05 \\
I axis & 63 & - & & 0.343 \\
II axis & 20.5714 & - & Rolling & Outer \\
\hline & & & 4.9 & 19.7 \\
Bearing 1 & 63 & $D_{\mathrm{P}}=610, d=62, n=42$ & 2.9 & 2.7 \\
Bearing 2 & 63 & $D_{\mathrm{P}}=692, d=118, n=53$ & 3.9 \\
Bearing 3 & 20.5714 & $D_{\mathrm{P}}=610, d=62, n=42$ & 32.6 \\
Bearing 4 & 20.5714 & $D_{\mathrm{P}}=692, d=118, n=53$ & 0.9 & 7.9 \\
\hline
\end{tabular}

spectrum, and envelope spectrum as shown in Figures 9(a), 9(b), and 9(c), respectively. Due to the strong background noise, we were unable to clearly distinguish the fault characteristics and the specific location of the damaged components. In order to find the specific location of the fault from the strong background noise, the proposed TFMSR method is used to process the signal. The obtained parameters are $\sigma$ $=1.0691, K=0.1048$, and $m=0.8175$. Figures $10(\mathrm{a})$ and $10(\mathrm{~b})$ show the time domain waveform and the spectrum obtained by the acquired signal. Figure 10(b) shows that the fault characteristic frequency is $25 \mathrm{~Hz}$. By comparing the characteristic frequency calculated in Table 1, we can see the characteristic frequency of the bearing 1 . The characteristic frequency of the inner ring is $24.3 \mathrm{~Hz}$. Therefore, we boldly conclude that the inner ring of the bearing 1 has failed.

Further, we use CBSR method to process the signal. The obtained parameters are $a=0.0708$ and $b=0.195$.
Figures 10(c) and 10(d) show the time domain waveform and the spectrum of the output signal. Figure $10(\mathrm{~d})$ shows that the fault characteristic frequency is $27.6 \mathrm{~Hz}$. By comparing with the data in Table 1, we cannot find the characteristic frequency which is close to $27.6 \mathrm{~Hz}$. This further proves the advancement of the proposed TFMSR method. To finally confirm the fault location, we use the endoscope to check the inside of the gearbox. It was finally confirmed that cracks occurred in the inner ring of the bearing 1, and the crack is shown in Figure 11. This is in complete agreement with our speculation, further validating the usability of the TFMSR method.

From the above analysis, we can conclude that the TFMSR method has a significant detection effect at 1-speed single faults. However, the availability of this method has yet to be verified in the face of weak fault detection under highspeed and multifault conditions. This will also be our next research work. 


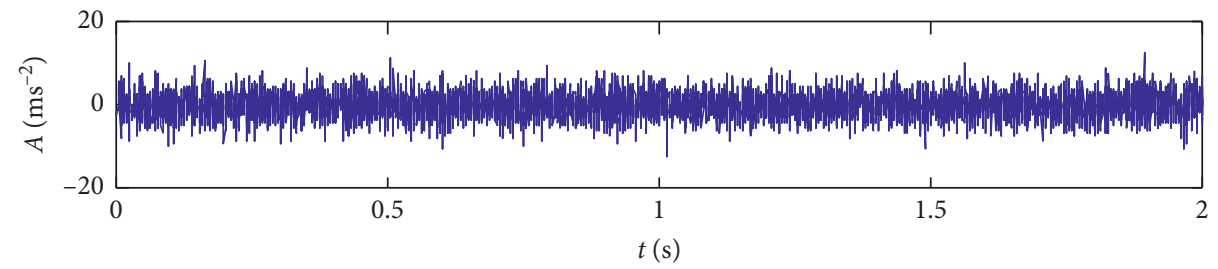

(a)

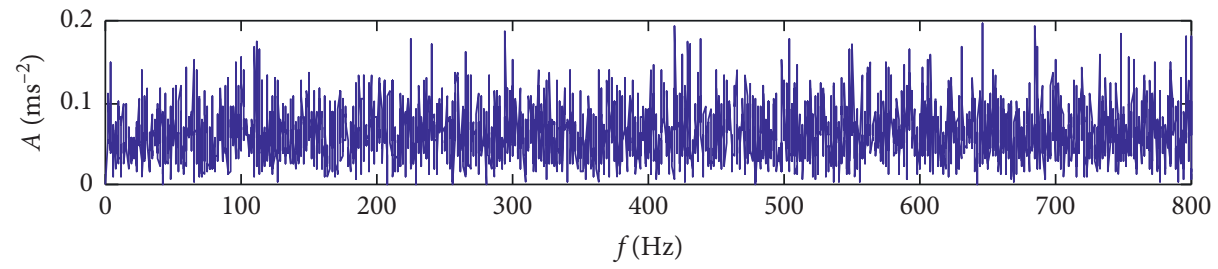

(b)

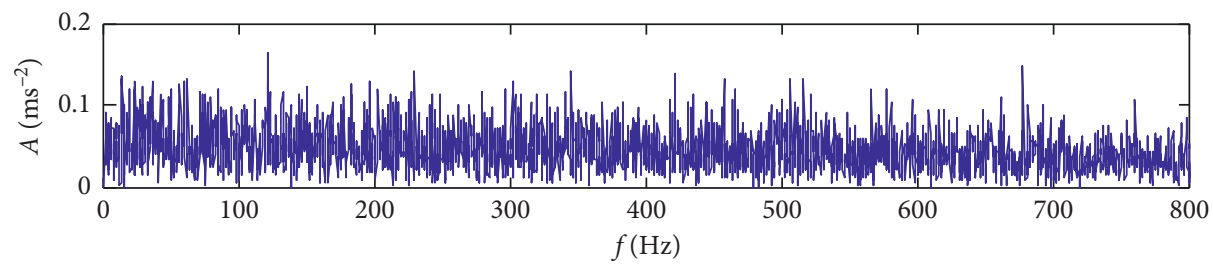

(c)

FIGURE 9: Rolling bearing signal: (a) original signal; (b) spectrum; (c) envelope spectrum.

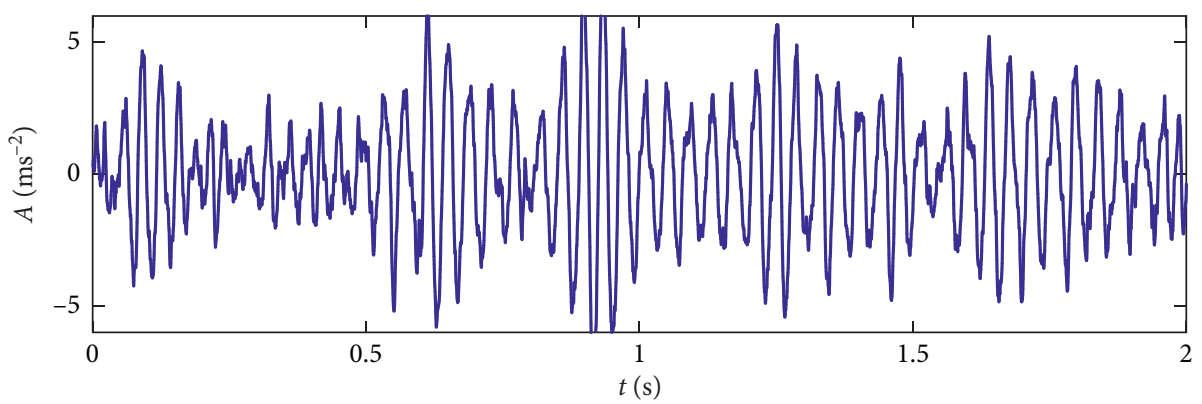

(a)

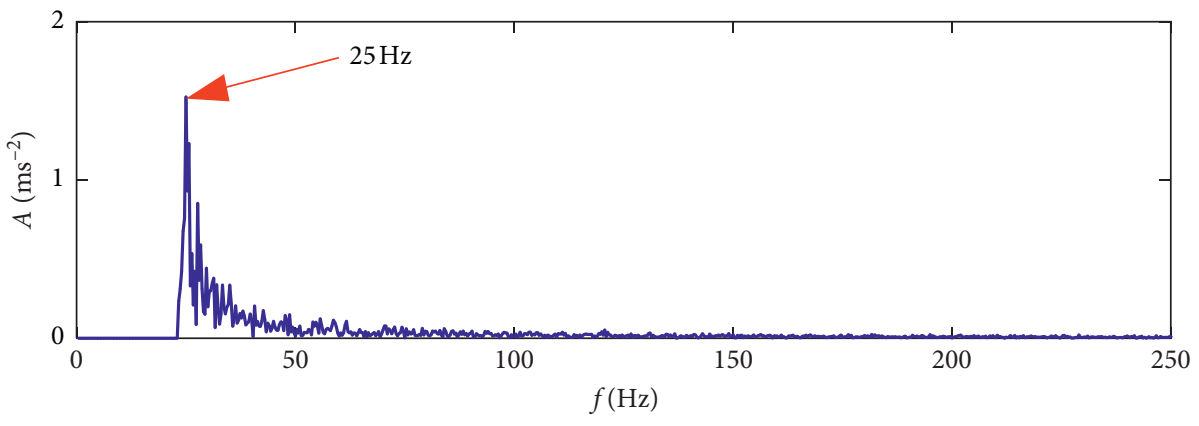

(b)

Figure 10: Continued. 


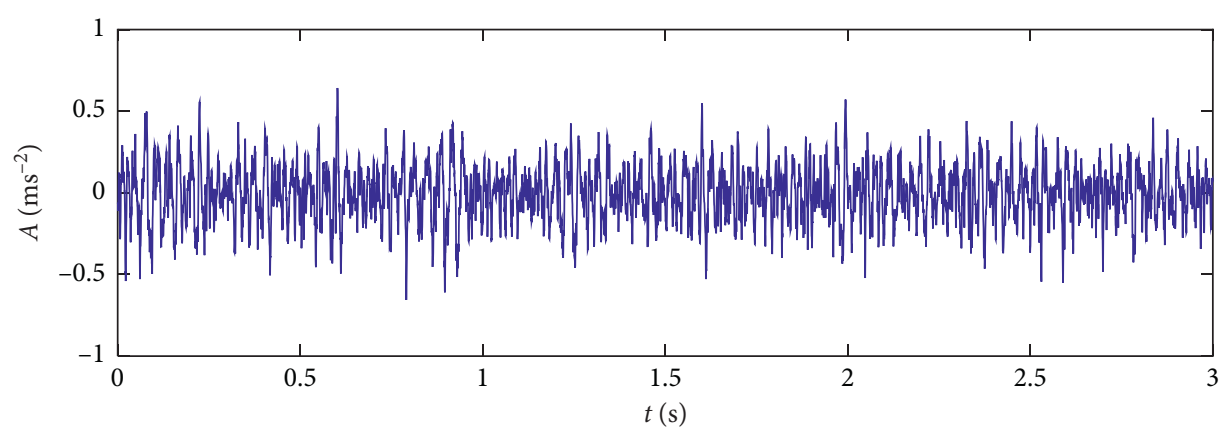

(c)

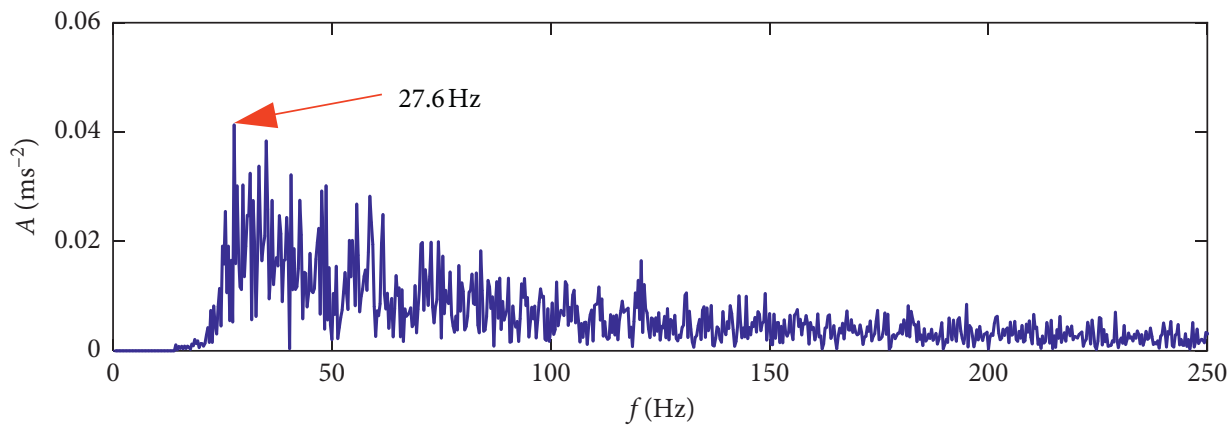

(d)

FIgURE 10: The results of the TFMSR method: (a) time domain waveform of the original signal; (b) spectrum. The results of the CBSR method: (c) time domain waveform of the original signal; (d) spectrum.

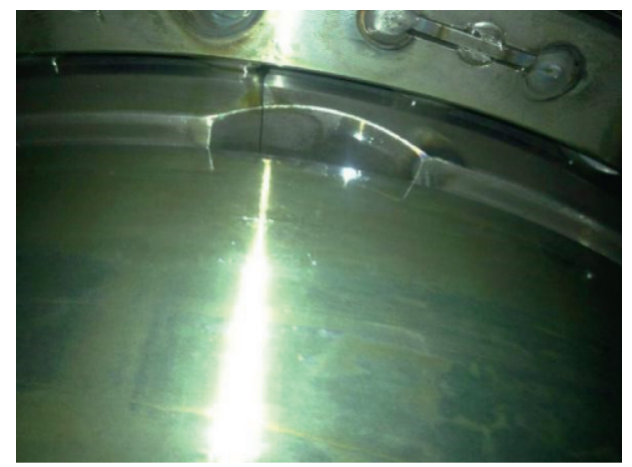

Figure 11: Gearbox bearing 1 with crack failure.

\section{Conclusion}

Considering the saturation characteristics of the CBSR model, this paper explores a new potential function model. Because of the short memory characteristics of the CBSR method, we add historical information to the feedback process of the SR. Through the combination of the above two methods, the weak fault extraction under strong background noise is realized. Based on the theoretical analysis and experimental results, the main conclusions are as follows:

(1) A potential function model with antisaturation characteristics is proposed, and the characteristics of the potential function model are analyzed.

(2) Considering the short memory characteristics of the CBSR method, a delay term and a feedback term are introduced on the basis of the proposed potential function model, and the output SNR is theoretically derived. The analysis of the effect of parameters on the output SNR is completed.

(3) The proposed method is used to simulate bearing fault extraction. Simulated results show that the proposed method effectively extracts fault features and is superior to the CBSR method.

(4) The proposed TFMSR method was used to verify the failure of the rolling mill gearbox. The experimental results show that the TFMSR method has better antisaturation characteristics and feedback characteristics than the CBSR method in extracting weak fault characteristics.

\section{Data Availability}

The data used to support the findings of this study are available from the corresponding author upon request.

\section{Conflicts of Interest}

The authors declare that they have no conflicts of interest.

\section{Acknowledgments}

This work was supported in part by the National Natural Science Foundation of China (51805275) and in part by the Beijing University of Science and Technology Innovation Talents Fund. 


\section{References}

[1] R. B. Randall and J. Antoni, "Rolling element bearing diagnostics-a tutorial," Mechanical Systems and Signal Processing, vol. 25, no. 2, pp. 485-520, 2011.

[2] N. Baydar, Q. Chen, A. Ball, and U. Kruger, "Detection of incipient tooth defect in helical gears using multivariate statistics," Mechanical Systems and Signal Processing, vol. 15, no. 2, pp. 303-321, 2001.

[3] N. Hu, M. Chen, G. Qin, L. Xia, Z. Pan, and Z. Feng, "Extended stochastic resonance (SR) and its applications in weak mechanical signal processing," Frontiers of Mechanical Engineering in China, vol. 4, no. 4, pp. 450-461, 2009.

[4] S. Yan, B. Ma, C. Zheng, and J. Chen, "An optimal lubrication oil replacement method based on selected oil field data," IEEE Access, vol. 7, pp. 92110-92118, 2019.

[5] R. Benzi, A. Sutera, and A. Vulpiana, "The mech-anism of stochastic resonance," Journal of Physics A: Mathematical and General, vol. 14, no. 11, pp. 453-457, 1981.

[6] R. Yan, R. X. Gao, and X. Chen, "Wavelets for fault diagnosis of rotary machines: a review with applications," Signal Processing, vol. 96, pp. 1-15, 2014.

[7] J. Lin and L. Qu, "Feature extraction based on morlet wavelet and its application for mechanical fault diagnosis," Journal of Sound and Vibration, vol. 234, no. 1, pp. 135-148, 2000.

[8] J. Chen, Z. Li, J. Pan et al., "Wavelet transform based on inner product in fault diagnosis of rotating machinery: a review," Mechanical Systems and Signal Processing, vol. 70-71, pp. 1-35, 2016.

[9] Y. Lei, J. Lin, Z. He, and M. J. Zuo, "A review on empirical mode decomposition in fault diagnosis of rotating machinery," Mechanical Systems and Signal Processing, vol. 35, no. 12, pp. 108-126, 2013.

[10] S. Zhang, Y. Wang, S. He, and Z. Jiang, "Bearing fault diagnosis based on variational mode decomposition and total variation denoising," Measurement Science and Technology, vol. 27 , no. 7, 2016.

[11] C. Cempel, "Generalized singular value decomposition in multidimensional condition monitoring of machines-A proposal of comparative diagnostics," Mechanical Systems and Signal Processing, vol. 23, no. 3, pp. 701-711, 2009.

[12] N. T. Thanh, P. Niamsup, and V. N. Phat, "Finite-time stability of singular nonlinear switched time-delay systems: a singular value decomposition approach," Journal of the Franklin Institute, vol. 354, no. 8, pp. 3502-3518, 2017.

[13] D. Huang, J. Yang, D. Zhou, M. A. F. Sanjuán, and H. Liu, "Recovering an unknown signal completely submerged in strong noise by a new stochastic resonance method," Communications in Nonlinear Science and Numerical Simulation, vol. 66, pp. 156-166, 2019.

[14] L. Gammaitoni, "The long run of the stochastic resonance idea," Journal of Physics A: Mathematical and Theoretical, vol. 49 , no. $45,2016$.

[15] A. Flores, S. Manilla, N. Huidobro et al., "Stochastic resonance in the synaptic transmission between hair cells and vestibular primary afferents in development," Neuroscience, vol. 322, pp. 416-429, 2016.

[16] J. Tang, B. Shi, and Z. Li, "Asymmetric delay feedback stochastic resonance detection method based on prior knowledge particle swarm optimization," Chinese Journal of Physics, vol. 56, no. 5, pp. 2104-2118, 2018.

[17] B. E. Klamecki, "Use of stochastic resonance for enhancement of low-level vibration signal components," Mechanical Systems and Signal Processing, vol. 19, no. 2, pp. 223-237, 2005.
[18] J. Li and X. Pan, "A nonlinear monostable filter for bipolar pulse signal detection," Mechanical Systems and Signal Processing, vol. 21, no. 3, pp. 1223-1232, 2007.

[19] Y. G. Leng, Y. S. Leng, T. Y. Wang, and Y. Guo, "Numerical analysis and engineering application of large parameter stochastic resonance," Journal of Sound and Vibration, vol. 292, no. 3-5, pp. 788-801, 2006.

[20] J. Tan, X. Chen, J. Wang et al., "Study of frequency-shifted and re-scaling stochastic resonance and its application to fault diagnosis," Mechanical Systems and Signal Processing, vol. 23, no. 3, pp. 811-822, 2009.

[21] J. Li, X. Chen, Z. Du, Z. Fang, and Z. He, "A new noisecontrolled second-order enhanced stochastic resonance method with its application in wind turbine drivetrain fault diagnosis," Renewable Energy, vol. 60, pp. 7-19, 2013.

[22] Y. Lei, D. Han, J. Lin, and Z. He, "Planetary gearbox fault diagnosis using an adaptive stochastic resonance method," Mechanical Systems and Signal Processing, vol. 38, no. 1, pp. 113-124, 2013.

[23] Q. He, J. Wang, Y. Liu, D. Dai, and F. Kong, "Multiscale noise tuning of stochastic resonance for enhanced fault diagnosis in rotating machines," Mechanical Systems and Signal Processing, vol. 28, pp. 443-457, 2012.

[24] S. Lu, Q. He, F. Hu, and F. Kong, "Sequential multiscale noise tuning stochastic resonance for train bearing fault diagnosis in an embedded system," IEEE Transactions on Instrumentation and Measurement, vol. 63, no. 1, pp. 106-116, 2014.

[25] P. Shi, H. Xia, D. Han, and R. Fu, "Dynamical complexity and stochastic resonance in an asymmetry bistable system with time delay," Chinese Journal of Physics, vol. 55, no. 1, pp. 133-141, 2017.

[26] Z. Li and B. Shi, "Fault diagnosis of rotating machinery based on stochastic resonance with a bistable confining potential," Shock and Vibration, vol. 2018, Article ID 8452509, 12 pages, 2018.

[27] K. Nishiguchi and A. Fujiwara, "Detecting signals buried in noise via nanowire transistors using stochastic resonance," Applied Physics Letters, vol. 101, no. 19, Article ID 193108, 2012.

[28] W. Zhao, J. Wang, and L. Wang, "The unsaturated bistable stochastic resonance system," Chaos: An Interdisciplinary Journal of Nonlinear Science, vol. 23, no. 3, Article ID 33117, 2013.

[29] M. Gosak, M. Perc, and S. Kralj, "Stochastic resonance in a locally excited system of bistable oscillators," The European Physical Journal B, vol. 80, no. 4, pp. 519-528, 2011.

[30] G. Zhang, D. Hu, and T. Zhang, "Stochastic resonance in unsaturated piecewise nonlinear bistable system under multiplicative and additive noise for bearing fault diagnosis," IEEE Access, vol. 7, pp. 58435-58448, 2019.

[31] M. L. Lv, G. Shen, and H. L. Wang, "Is the high-frequency signal necessary for the resonance in the delayed system," Chinese Physics Letters, vol. 32, no. 1, pp. 15-17, 2015.

[32] J. H. Yang, M. A. F. Sanjuán, and H. G. Liu, "Signal generation and enhancement in a delayed system," Communications in Nonlinear Science, vol. 22, no. 1-3, pp. 1158-1168, 2015.

[33] P. Zhuo, Y. Zhu, W. Wu, J. Shu, and T. Xia, "Real-time Fault diagnosis for gas turbine blade based on output-hidden feedback elman neural network," Journal of Shanghai Jiaotong University (Science), vol. 23, no. S1, pp. 95-102, 2018.

[34] T. Xia, Y. Song, Y. Zheng, E. Pan, and L. Xi, "An ensemble framework based on convolutional bi-directional LSTM with multiple time windows for remaining useful life estimation," Computers in Industry, vol. 115, Article ID 103182, 2020. 
[35] S. Guillouzic, I. L'Heureux, and A. Longtin, "Small delay approximation of stochastic delay differential equations," Physical Review E, vol. 59, no. 4, pp. 3970-3982, 1999.

[36] T. D. Frank, "Delay Fokker-Planck equations, perturbation theory, and data analysis for nonlinear stochastic systems with time delays," Phys Rev E Stat Nonlin Soft Matter Phys, vol. 71, no. 3, p. 31106, 2005

[37] B. Mcnamara and K. Wiesenfeld, "Theory of stochastic resonance," Physical Review A, vol. 39, no. 9, pp. 4854-4869, 1989.

[38] S. Guillouzic, I. L'Heureux, and A. Longtin, "Small delay approximation of stochastic delay differential equations," Physical Review E, vol. 59, no. 4, pp. 3970-3982, 1999.

[39] S. D. Nguyen, Q. H. Nguyen, and S.-B. Choi, "A hybrid clustering based fuzzy structure for vibration control-part 2: an application to semi-active vehicle seat-suspension system," Mechanical Systems and Signal Processing, vol. 56-57, pp. 288-301, 2015.

[40] B. Øksendal, Stochastic Differential Equations, Springer, Berlin, Germany, 2003. 